\title{
Correction to: Demonstration of entanglement purification and swapping protocol to design quantum repeater in IBM quantum computer
}

\author{
Bikash K. Behera ${ }^{1}$ - Swarnadeep Seth ${ }^{1,2} \cdot$ Antariksha Das $^{1,3}$. \\ Prasanta K. Panigrahi ${ }^{1}$ \\ Published online: 19 March 2019 \\ ○) Springer Science+Business Media, LLC, part of Springer Nature 2019
}

\section{Correction to: Quantum Information Processing (2019) 18:108 https://doi.org/10.1007/s11128-019-2229-2}

The article Demonstration of entanglement purification and swapping protocol to design quantum repeater in IBM quantum computer, written by Bikash K. Behera, Swarnadeep Seth, Antariksha Das, Prasanta K. Panigrahi, was originally published electronically on the publisher's internet portal (currently SpringerLink) on 1 March 2019 with open access.

With the author(s)' decision to step back from Open Choice, the copyright of the article changed on 19 March 2019 to (C) Springer Science+Business Media, LLC, part of Springer Nature 2019 and the article is forthwith distributed under the terms of copyright.

The original article has been corrected.

The original article can be found online at https://doi.org/10.1007/s11128-019-2229-2.

Prasanta K. Panigrahi

pprasanta@iiserkol.ac.in

Bikash K. Behera

bkb18rs025@iiserkol.ac.in

Swarnadeep Seth

swarnadeep@knights.ucf.edu

Antariksha Das

a.das-1@tudelft.nl

1 Department of Physical Sciences, Indian Institute of Science Education and Research Kolkata, Mohanpur, West Bengal 741246, India

2 Department of Physics, University of Central Florida, Orlando, FL 32186, USA

3 QuTech, Delft University of Technology (TU Delft), Lorentzweg 1, 2628 CJ Delft,

The Netherlands 
Publisher's Note Springer Nature remains neutral with regard to jurisdictional claims in published maps and institutional affiliations. 